\title{
O GÊNERO HOLOCHILUS (MAMMALIA: CRICETIDAE) NO RIO GRANDE DO SUL: TAXONOMIA E DISTRIBUIÇÃO
}

Rosane Vera Marques

\begin{abstract}
The genus Holochilus Brandt, 1835 is represented in Rio Grande do Sul, Southern Brazil, by two forms: H. brasiliensis vulpinus (Brants, 1827) e H. magnus Hershkovitz, 1955. They are myomorph rodents with semi-aquatic habits. Species of Holochilus range from Northern South America to Central Argentina.

In this paper, I present an analysis of standard measurements of specimens collected in Southern Brazil and deposited in the collections of $F Z B, P U C$ and UFRGS and a distribution map with collecting localities.
\end{abstract}

\section{INTRODUÇÃO}

O gênero Holochilus é constituído por cricetídeos de vida semiaquática, distribuídos desde a Venezuela e Guianas até a Argentina Central e o Uruguai. Segundo Hershkovitz, 1955, o gênero está constituído por duas espécies, $H$. brasiliensis, com várias subespécies e com distribuição semelhante à do gênero, e $H$. magnus com distribuiç̧-ao restrita ao Uruguai e sul do Brasil. Contudo, outros autores, mesmo após a publicação da revisão feita por Hershkovitz, ainda consideram válidas as espécies $H$. sciureus (Twigg, 1962 e 1965; Ozanan, 1969 e Massoia, 1981; que ocorreria na porção mais setentrional da América do Sul) e $H$. chacarius /Vidal et alii, 1976 e 1978; Massoia, 1976 (comunicação pessoal e Vidal et alii, 1976) que habitaria a região entre o sul do Paraguai e o noroeste da Argentina].

Tate, 1932 publicou a história taxonômica de alguns gêneros de cricetídeos neotropicis, estando Holochilus incluído entre eles. O primeiro exemplar de Holochilus foi coletado por Saint-Hilaire e descrito como espécie nova sob o nome de Mus brasiliensis por Desmarest, 1819. Brants, 1827 descreveu Mus vulpinus e Mus physodes. Brandt, 1835 propôs o nome Holochilus como um subgênero de Mus e descreveu a nova espécie Mus (Holochilus) leucogaster; Wagner, 1842 tratou Holochilus como um gênero distinto e descreveu uma nova espécie, $H$. sciureus. 
Burmeister, 1854 considerou Holochilus como subgênero de Hesperomys, incluindo Nectomys em Holochilus. Lilljeborg, 1866 considerou Holochilus como um gênero distinto de Hesperomys, mas continuava a incluir Nectomys em Holochilus, sendo que essa idéia foi apoiada por Thomas, 1884 e 1896. Porém, Thomas, 1897 acabou por estabelecer a distinção entre os gêneros Holochilus e Nectomys. Hershkovitz, 1944 escreveu sobre algumas semelhanças e diferenças entre os dois gêneros. Várias espécies foram descritas já sob a denominação genérica de Holochilus: H. nanus Thomas 1897; H. guianae Thomas, 1901; H. venezuelae Allen, $1904 ; H$. chacarius e $H$. balenearum Thomas, 1906; H. amazonicus Osgood, 1915; H. incarum Thomas, 1921. H. multannus do Pleistoceno Superior foi descrito por Ameghino, 1889 como uma espécie extinta, mas foi considerada idêntica a $H$. brasiliensis vulpinus por Hershkovits, 1955. Hershkovits, 1955 reuniu todas as espécies descritas de Holochilus em uma espécie única, $H$. brasiliensis, reconhecendo várias subespécies. A outra espécie, $H$. magnus, foi descrita nesse mesmo trabalho, sendo que o autor tomou como tipo um espécimen pertencente ao Chicago Natural History Museum que havia sido coletado por Sanborn em 1926 em "Passo de las Avarias" ("Paso de Averías" fide Hershkovit), rio Cebollati na República do Uruguai e que havia sido identificado como H. vulpinus por Sanborn, 1929.

Os nomes populares dos ratos do gênero Holochilus diferem de um local para outro. São chamados de rato de cana (Moojen, 1943; Ozanan, 1969) no nordeste do Brasil; ratão da taquara (Giovannoni et alii, 1946) em "ratadas" no planalto paranaense; rato do junco no Rio Grande do Sul (comunicação pessoal do laboratorista E. Borsatto da Fundação Zoobotânica do Rio Grande do Sul); rata de água (Ximenez et alii, 1972) no Uruguai; rata nutria (Massoia, 1971) ou colorada (Lucero, 1983) na Argentina; Angudyá pihtá em guarani, segundo Dennler, 1939 (Angudyá $=$ rato; pihtá $=$ vermelho).

Hershkovitz, 1955 reuniu o gênero Holochilus juntamente com Sigmodon, Reithrodon e Neotomys no grupo dos sigmodontes. Hooper \& Musser, 1964, estudando a morfologia peniana de roedores, relacionaram Holochilus com Oryzomys por causa da semelhança apresentada no pênis entre esses dois gêneros. Hershkovitz, 1966 escreveu que não é possível tomar como provas de parentesco entre dois gêneros apenas a similaridade do falus que possa existir entre eles. Carleton, 1973, estudando a morfologia do estômago de cricetídeos, observou que havia semelhanças na distribuição de epitélio gástrico glandular entre os quatro gêneros de sigmodontes considerados por Hershkovitz, mas que essas semelhanças eram estendidas também a outros gêneros como Euneomys e Nectomys.

Estudos sobre o cariótipo de Holochilus realizados por Gardner \& Patton, 1976; Vidal et alii, 1976 e 1978, Riva et alii, 1977 e Freitas, 1983 demonstraram que se trata de um padrão complexo que pode apresentar 
polimorfismo cromossômico intrapopulacional, ocorrendo fusões robertsonianas, inversões pericêntricas e apresentando um par de pequenos cromossomos metacêntricos considerados como "marcadores" do gênero.

Os ratos do gênero Holochilus alimentam-se de plantas herbáceas tenras e aquáticas, podendo incluir em sua dieta moluscos, crustáceos (Devincenzi, 1935 e Sierra \& Achaval, 1971) e, eventualmente, besouros (Twigg, 1962) e aranhas e grilos (Barlow, 1969). Atraídos pela abundância de alimento e refúgio, além de proximidade com água, os ratos desse gênero costumam freqüentar cultivos de cana-de-açúcar nas baixadas (Ozanan, 1969; Massoia, 1974; Twigg, 1962), bem como arrozais alagados; eventualmente, podem atacar plantações de milho, de algodão (Ozanan, 1969) e até bananais e hortas (Massoia, 1974). Foi constatada a presença de indivíduos desse gênero em "ratadas" (Giovannoni et alii, 1946; Llanos, 1944); após o consumo de todo o alimento produzido na frutificação de taquarais, os roedores costumam invadir terrenos cultivados, assim como paióis e até residências.

Sendo um animal de hábitos semi-aquáticos, Holochilus é um bom reservatório de Schistosoma mansoni, não apenas podendo ser hospedeiro dos parasitas adultos, mas também eliminando ovos maduros em suas fezes (Dias, Ávila- Pires \& Pinto, 1978). Carvalho, Andrade \& Cortes, 1976 consguiram fechar o ciclo do $S$. mansoni sem direta participação humana em condições semi-naturais, utilizando indivíduos de $\mathrm{H}$. brasiliensis sãos e Biomphalaria glabrata infectadas com uma cepa humana. Holochilus ainda pode ser parasitado por Trypanosoma cruzi (Barreto \& Ribeiro, 1974). Twigg, 1965b constatou parasitismo de dípteros da espécie Cuterebra apicalis sobre esses roedores na Guiana Inglesa; esse mesmo autor, em outro trabalho do mesmo ano, realizou estudos ecológicos com população pertencente a esse gênero.

No Rio Grande do Sul, o gênero está representado por duas espécies; $H$. brasiliensis vulpinus (Brants, 1827) que tem como localidade tipo a margens do Rio Uruguai em território rio-grandense (Hershkovits, 1955), e H. magnus Hershkovits, 1955 coletado pela primeira vez no Brasil no ano de 1979, nas margens do Rio Ivaí, no município de Tupanciretã.

\section{MATERIAL UTILIZADO}

Foi utilizado material taxidermizado ou conservado em líquido das seguintes coleções científicas: Museu de Ciências Naturais da Fundação Zoobotânica do Rio Grande do Sul (MCN); Coleção de Mamíferos da Pontifícia Universidade Católica (CMPUC); e também foram estudados exemplares que ainda não deram entrada em coleção de nenhuma insti- 
tuição, permanecendo com os respectivos números de campo: Luís Flamarion (LF); Mamífero (M).

O material examinado foi o seguinte:

Holochilus brasiliensis vulpinus: Fazenda Chaleira Preta, Montenegro, RS 12/VIII/1977: MCN.626 e MCN.689; Morro do Pesqueiro, Montenegro, RS 10/VI/1977: MCN.594; BR290 km123 Pântano Grande, Rio Pardo, RS - 11/IX/1977: MCN.658 a MCN.672; Banhado do Pontal, Triunfo, RS - 16/XI/1975: MCN.326; Fazenda da Taipa, Rosário do Sul, Rs - 09-10/IX/1977: MCN.645, MCN.646 e MCN.651; Taim, Rio Grande, RS - 06/VII/1976: LF.102; Passo do S, São Francisco de Paula, RS 05/VII/1981: CMPUC.020; Banhado do Alemão (UFPEL), Pelotas, RS - VII/1981: CMPUC.0170 a CMPUC.0173, CMPUC.0214, M.1007, M.1005 e M.1010; sem procedência: LF.002.

Holochilus magnus: margem do Rio Ivaí, tupanciretã, RS - I/1979: LF.081, LF.084, LF.095, LF.155, LF.087.

\section{MÉTODO}

Após a identificação dos exemplares, o trabalho consistiu, basicamente, de três etapas principais que foram: medições cranianas, desenhos de séries molares e elaboração de um mapa da distribuição do gênero Holochilus para o Rio Grande do Sul.

Os crânios foram medidos com o auxílio de um paquímetro Mitutoyo com a precisão de $0,02 \mathrm{~mm}$. As medidas realizadas foram as seguintes: CTC = comprimento total do crânio; $\mathrm{CCB}=$ comprimento côndilo-basal; LZ = largura zigomática; LMI = largura mínima interobital; $\mathrm{CSM}=$ comprimento da série molar superior esquerda (alvéolo).

Para as medidas do corpo, foram utilizadas as medidas realizada em campo contidas no livro ou fichas de registro de coleção dos museus, sendo as seguintes: $\mathrm{CT}=$ comprimento total; $\mathrm{CC}=$ comprimento de cau$\mathrm{da} ; \mathrm{CCC}^{*}=$ comprimento da cabeça e corpo; PSU = comprimento do pé posterior sem unha; $\mathrm{PCU}=$ comprimento do pé posterior com unha; $\mathrm{CO}=$ comprimento da orelha. (*Calculado subtraindo-se o CC do CT.)

Os desenhos das séries molares foram feitos com a utilização de câmara clara acoplada a um miscroscópio estereoscópico Wild M-3 com aumento de 10/21 X 16 .

O mapa de distribuição do gênero Holochilus no Rio Grande do Sul foi feito com base nos dados de livro ou fichas de registro de coleções ou das etiquetas das peles e daqueles constantes em relatórios da Fundação Zoobotânica do Rio Grande do Sul (Delta do Jacuí e Barragem Saturnino de Brito). A observação direta de animais vivos na Estação Experimental do IRGA (Instituto Rio-grandense do Arroz) no município de Cachoeirinha durante os meses de dez/1983 e jan a fev/1984 também foi considerada válida. Como base, foi utilizado um mapa mudo da região sul do Brasil com escala de 1:5.000.000 editado pelo Ministério da Educação e Cultura. Foram examinadas cartas geográficas elaboradas pelo 
exército do Brasil para determinação das coordenadas geográficas das localidades nas quais foi constatada a presença do gênero Holochilus, sendo assinaladas no mapa mudo.

\section{COMENTÁRIOS}

Segundo Hershkovitz, 1955, as principais características para a diferenciação entre as espécies $H$. magnus e $H$. brasiliensis são:

H. magnus

compr. cauda (CC)

pé post. c/u

forâmen incisivo

$\mathrm{M}^{3}$ (terceiro molariforme superior) maior que $210 \mathrm{~mm}$ e mais longo que compr. cabeça e corpo (CCC)

maior que $48 \mathrm{~mm}$

extendido posteriormente até o plano anterior dos primeiros molares ou ultrapassando-o

tâo longo quanto largo mais curto que $\mathrm{M}^{2}$ (segundo molariforme sup.)

\section{H. brasiliensis}

menor que $210 \mathrm{~mm}$ e igual ou menor que compr. cabeça e corpo (CCC)

menor que $48 \mathrm{~mm}$

não extendido posteriormente até $\circ$ plano anterior dos primeiros molares

mais longo que largo, mais longo que $\mathrm{M}^{2}$ (segundo molariforme superior)

As medidas realizadas demonstram que, nem sempre, o comprimento da cauda de $H$. brasiliensis é menor do que $210 \mathrm{~mm}$ e que o comprimento da cabeça e corpo pode ser menor que o da cauda, mas que, realmente, essa diferença entre o comprimento da cabeça e corpo e da cauda é mais expressiva em $H$. magnus. Também é verificado que o comprimento do pé posterior com unha de $H$. brasiliensis pode ter um valor acima de $48 \mathrm{~mm}$.

As características cranianas, como as diferenças entre o forámen incisivo e os molariformes (ver estampas) das duas espécies, salientada por Hershkovitz, puderam ser verificadas, sendo possível concluir que são mais confiáveis como características distintivas entre as duas espécies do que as caracterśiticas do corpo.

Devido ao pequeno número de indivíduos da espécie $H$. magnus coletados até hoje e à pequena área de distribuição dessa espécie em relação à área de $H$. brasiliensis, surgiria a dúvida de que realmente se tratem de espécies distintas.

O mapa demonstra que poucas coletas foram feitas para que se possa ter uma idéia conclusiva sobre a distribuição das formas do gênero Holochilus no Rio Grande do Sul. Contudo, é possível notar que o gênero está presente em várias regiões fisiográficas do Estado, sendo bem provável sua presença em quase todo o território rio-grandense, desde que os biótopos utilizados por esse gênero, que são os de banhado e mar- 
gens de córregos e rios, se façam presentes. Além disso, todo o território ocupado por plantações de arroz pode ser considerado como propício à ocorrência de ratos do junco.

Tabela 1

Medidas cranianas de $H$. brasiliensis vulpinus (mm)

\begin{tabular}{lcccccc}
\hline \multicolumn{1}{c}{ No exemplar } & Sexo & CTC & CCB & LZ & LMI & CSM \\
\hline M. 1005 & $?$ & 34,2 & 32,2 & 20,2 & 4,5 & 7,72 \\
CMPUC. 0214 & $?$ & 35,5 & 34,0 & 21,4 & 4,4 & 7,42 \\
M. 1010 & $?$ & 36,1 & 34,3 & 20,8 & 4,5 & 7,96 \\
CMPUC. 0170 & $?$ & 36,4 & 34,4 & 21,8 & 4,5 & 7,60 \\
MCN. 594 & F & 36,5 & 34,6 & 21,6 & 4,5 & 8,04 \\
MCN. 672 & F & 37,4 & 35,4 & 21,8 & 4,5 & 7,66 \\
MCN. 664 & F & 38,6 & 37,4 & 22,0 & 4,7 & 7,82 \\
CMPUC. 0172 & ? & 39,5 & 32,2 & 22,3 & 4,9 & 8,10 \\
MCN. 660 & F & 39,7 & 37,4 & - & 4,9 & 7,52 \\
MCN. 662 & F & 39,7 & 38,5 & 23,2 & 5,2 & 7,84 \\
MCN. 659 & F & 39,7 & 38,6 & - & 4,6 & 7,86 \\
MCN. 663 & M & 40,0 & 39,3 & 23,7 & 4,5 & 7,98 \\
CMPUC. 020 & F & 40,1 & 38,4 & 23,7 & 4,6 & 8,00 \\
MCN. 670 & F & 40,5 & 39,3 & 23,6 & 4,4 & 8,04 \\
MCN. 668 & F & 40,7 & 38,5 & - & 4,6 & 8,22 \\
MCN. 667 & F & 41,1 & 38,8 & 24,1 & 4,7 & 7,86 \\
MCN. 666 & F & 41,2 & - & 23,8 & 4,4 & 7,86 \\
LF. 102 & $?$ & 42,8 & 40,7 & - & 4,7 & 8,14 \\
CMPUC. 0171 & $?$ & 43,5 & 41,4 & 25,1 & 4,7 & 7,76 \\
MCN. 651 & F & 44,2 & - & 25,7 & 5,2 & 8,52 \\
M. 1007 & $?$ & 44,5 & 43,4 & 26,0 & 5,1 & 7,54 \\
MCN. 661 & M & 44,8 & - & - & 5,1 & 8,06 \\
MCN. 326 & M & 47,5 & 44,3 & - & 5,1 & 7,68 \\
MCN. 658 & M & - & - & - & 5,0 & 8,04 \\
MCN. 665 & F & - & - & - & - & 8,06 \\
MCN. 669 & F & - & - & - & 4,8 & 7,98 \\
MCN. 671 & F & - & - & - & 4,6 & 8,24 \\
CMPUC. 0173 & $?$ & - & 37,4 & 23,3 & 4,5 & 7,56 \\
LF. 002 & $?$ & - & - & 21,2 & 4,7 & 7,72 \\
\hline
\end{tabular}

CTC: comprim. total do crânio; CCB: comprim. côndilo-basal; LZ: largura zigomática; LMI: largura mínima interorbital; CSM: comprim. da série molar superior esquerda. (-) medida não realizada devido ao fato de alguns crânios estarem quebrados.

Os principais produtores de arroz no Rio Grande do Sul, segundo o Ministério do Interior - SUDESUL, 1978, são os municípios de Uruguaiana, Dom Pedrito, São Gabriel, Alegrete, Bagé, Rosário do Sul na região da Campanha; Camaquã, Arroio Grande, Jaguarão, Tapes, São Lourenço do Sul, Pedro Osório, Pelotas, na Encosta do Sudeste; Cachoeira do Sul, São Sepé, Rio Pardo, Cacequi, na Depressão Central; Santa Vitória do Palmar, Osório, no Litoral; e Itaqui e São Borja, nas 


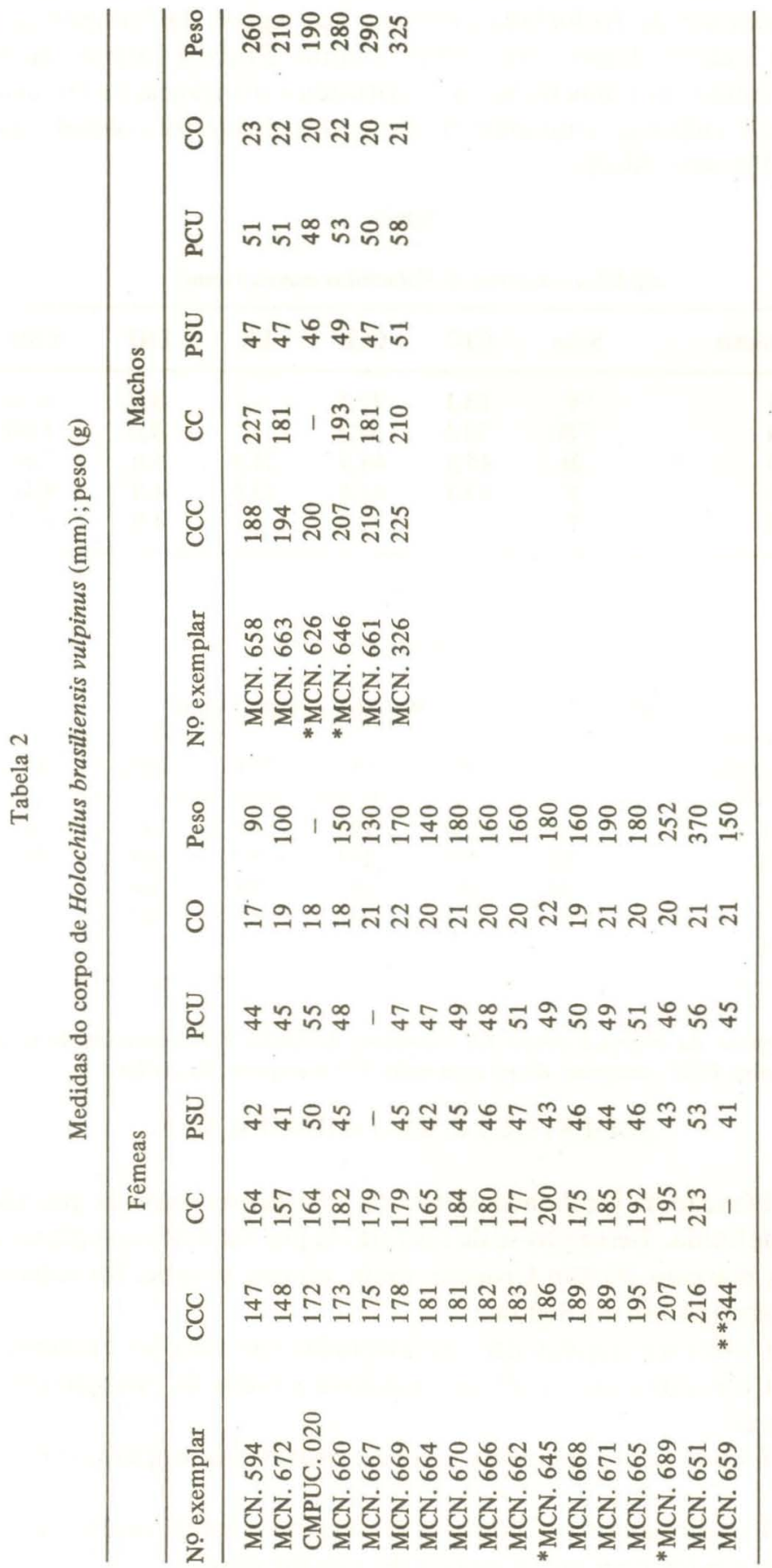


Missões. Dentre essas regiões, apenas a das Missões não teve ainda nenhum exemplar de Holochilus coletado; nas regiões da Campanha, Encosta do Sudeste, Depressão Central, Litoral, Encosta Inferior do Nordeste e Campos de Cima da Serra é verificada a ocorrência de Holochilus brasiliensis vulpinus; enquanto $H$. magnus somente foi coletado na região do Planalto Médio.

Tabela 3

Medidas cranianas de Holochilus magnus (mm)

\begin{tabular}{lcccccc}
\hline No exemplar & Sexo & CTC & CCB & LZ & LMI & CSM \\
\hline LF. 081 & $*$ F & 35,1 & 32,5 & - & 5,0 & 8,12 \\
LF. 084 & ${ }^{*}$ M & 35,6 & 32,9 & - & 5,0 & 8,00 \\
LF. 087 & M & 45,5 & 44,3 & 25,9 & 5,0 & 7,84 \\
LF. 095 & F & 43,5 & 41,8 & 23,5 & 4,8 & 8,16 \\
LF. 155 & $?$ & - & 32,5 & - & 4,9 & 8,12 \\
\hline
\end{tabular}

* Jovem

Tabela 4

Medidas do corpo de Holochilus magnus (mm)

\begin{tabular}{lcccccc}
\hline No exemplar & Sexo & CCC & CC & PSU & PCU & CO \\
\hline LF. 081 & ${ }^{*} \mathrm{~F}$ & 134 & 191 & 46 & 50 & 20 \\
LF. 084 & ${ }^{* M}$ & 141 & 203 & 50 & 53 & 21 \\
LF. 087 & M & 211 & 253 & 59 & 64 & 25 \\
LF. 095 & $* *$ F & 196 & 260 & 58 & 62 & 22,5 \\
LF. 155 & $?$ & - & - & - & - & - \\
\hline
\end{tabular}

* jovem; ** grávida

CCC: comprim. da cabeça e corpo; CC: comprim. da cauda; PSU: comprim. do pé posterior sem unha; $\mathrm{PCU}$ : comprim. do pé com unha; $\mathrm{CO}$ : comprim. da orelha.

\section{SUGESTÕES E RECOMENDAÇÕES}

1. Fixação da localidade tipo de $H$. brasiliensis vulpinus que não está bem definida. Deixando-se de restringi-la por falta de exemplares coletados às margens do Rio Uruguai, onde, ao que se sabe, foi coletado o espécimen tipo por F. Sellow.

2. Coleta nas regiões não representadas nas coleções existentes.

3. Coleta de séries de H. magnus para estudos de variação intrapopulacional.

4. Estudo sobre simpatria entre $H$. brasiliensis vulpinus e $H$. magnus.

5. Estudos sobre a ecologia de $H$. magnus e comparação com os estudos realizados com outra espécie do mesmo gênero. 
6. Cruzamento em cativeiro de indivíduos pertencentes às duas espécies para se chegar à conclusão definitiva de que sejam ou não realmente espécies diferentes.

\section{AGRADECIMENTOS}

Sinceros agradecimentos a: Fernando Dias de Ávila-Pires pela orientação do trabalho e leitura dos manuscritos. Stela Máris P. Gayer pela instrução na execução dos desenhos. Heinrich Hasenack pela instrução na elaboração do mapa. Jocélia Grazia pelo auxílio prestado na organização das estampas. Mara da Silveira Benfato, Marta Fabián, Lígia Krause e Maria Dalva Barbosa pela leitura dos manuscritos e sugestões. Vitor Hugo Travi da Pontifícia Universidade Católica do Rio Grande do Sul por permitir o acesso aos exemplares da coleção de mamíferos daquela Universidade. Museu de Ciências Naturais da Fundação Zoobotânica do Rio Grande do Sul pelo empréstimo dos exemplares pertencentes à coleção de mamíferos.

\section{BIBLIOGRAFIA}

Além das referências bibliográficas citada no texto, são indicados alguns trabalhos de interesse especial para o tema, assinalados com um asterisco.

*Achaval, F.; González, J. G.; Meneghel, M. \& Melgarejo, A. R., 1979. Lista comentada del material recogido en costas uruguayas, transportado por camalotes desde el río Paraná. Acta Zool. Lilloana, 35(1):195-200.

Allen, J. A., 1904. New mammals from Venezuela and Colombia. Bull. Am. Mus. Nat. Hist., 20(28):327-335.

*Allen, J. A., 1916. Mammals collected on the Roosevelt Brazilian Expedition, with field notes by Leo E. Miller. Bull. Am. Mus. Nat. Hist., 35(30): 559-610.

Ameghino, F., 1889. Contribucion al conocimiento de los mamiferos fosiles de la República Argentina. In: Obras completas y correspondencia cientifica de F. A. Dir. por A. J. Torcelli. Vol. 6 Buenos Aires, La Plata. 621 pp. 1916.

*Barbosa, F. S., 1972. Natural infection with Schistosoma mansoni in small mammals trapped in the course of schistosomiasis control project in Brazil. J. Parasitol., 58(2):405-407.

Barlow, J. C., 1969. Observations on the biology of rodents in Uruguay. Life Sci. Contr. Roy Ontario Mus., (17):1-59.

Barreto , M. P. \& Ribeiro, R.D., 1974. Estudos sobre reservatórios e vectores silvestres do Trypanosoma cruzi. 55. Infecção natural do rato Holochilus brasiliensis leucogaster (Brandt, 1835) pelo T. cruzi. Rev. Inst. Med. Trop. São Paulo, 16(5):270-275.

Brants, A., 1827. Het Geslacht der Muizen door Linnaeus opgesteld. Berlin. p. 137-138.

Carleton, M. D., 1973. A survey of gross stomach morphology in New World Cricetinae (Rodontia, Muroidea) with comments on functional interpretations. Misc. Publ. Mus.Zool. Univ. Mich., (146):1-43. 
Carvalho, O. S.; Andrade, R. M. \& Cortes, M. I. N., 1976. Ciclo vital de Schistosoma mansoni através do Holochilus brasiliensis (Desmarest, 1818), em ambiente seminatural (Trematoda, Schistosomatidae; Rodentia, Cricetidae). Rev. Soc. Bras. Med. Trop. 10(5):235-247.

*Cerqueira, R., 1975. Sobre a localidade tipo de Holochilus brasiliensis vulpinus (Brants, 1827) (Rodentia, Cricetidae). Rev. Bras. Biol., 35(1):31-34.

*Cerqueira, R. \& Klaczko, L. B., 1975. Biometric studies on Holochilus brasiliensis (Rodentia, Cricetidae). 1. Ontogenetic variation of a population at Crato, northeastern Brazil. Rev. Bras. Biol., 35(1):35-38.

Dennler, J.G., 1939. Los nombres indigenas en guarani. Physis (B. Aires), 16:225-244.

Devincenzi, G. J., 1935. Mamíferos del Uruguay. An. Mus. Hist. Nat. Montevideo, $4(10): 1-96$.

*Dias, L.C.S., 1973. Small wild mammals naturally infected with Schistosoma mansoni. Rev. Saúde Pública, 6(2):233.

Dias, L. C. S.; Ávila-Pires, F. D. \& Pinto, A. C. M., 1978. Parasitological and ecological aspects of schistosomiasis mansoni in the valley of the Paraíba do Sul River (São Paulo State, Brazil). 1. Natural infection of small mammals with Schistosoma mansoni. Trans. R. Soc. Trop. Med. Hyg., 72(2):496-500.

*Fornes, A. \& Massoia, E., 1965. Small mammals (Marsupialia and Rodentia) collected in the locality of Miramar, Province of Buenos Aires. Physis (B. Aires), 25(69):99-108.

Freitas, T. R. O. et al., 1983. Chromosome relationships in three representatives of the genus Holochilus (Rodentia, Cricetidae) from Brazil. Genetica, 61(1):13-30.

Fundação Zoobotânica do Rio Grande do Sul - Museu de Ciências Naturais, 1982. Estudo preliminar da flora e fauna na área da barragem Saturnino de Brito, CORSAN Santa Maria, RS. Porto Alegre. 70 pp.

Gardner, A. L. \& Patton, J. L. 1976. Karyotypic variation in oryzomyine rodents (Cricetinae) with comments on chromosomal evolution in the neotropical cricetine complex. Occas. Pap. Mus. Zool. La State Univ., (49):1-48.

Giovannoni, Vellozo \& Kubiak, 1946. Sobre as "ratadas" do primeiro planalto paranaense. Archos Biol. Tecnol., Curitiba, 1:185-195.

Hershkovitz, P., 1944. A systematic review of the neotropical water rats of the genus Nectomys (Cricetinae). Mus. Zool., (58):1-101.

Hershkovitz, P., 1955. South American marsh rats, genus :Holochilus, with a summary of sigmodont rodents. Fieldiana Zool. 37:639-687.

Hershkovitz, P., 1966a. South American swamp and fossorial rats of the Scapteromyine Group (Cricetinae, Muridae) with comments on the glans penis in murid taxonomy. Z. Saeugetier kd., 31(2):81-149.

Hooper, E. T. \& Musser, G.G., 1964. The glans penis in Neotropical Cricetines (Family Muridae) with comments on classification of Muroid Rodents. Misc. Publ. Mus. Zool. Univ. Mich., (123):1-57.

*Langguth, A., 1965. Contribución al conocimiento de los Cricetinae del Uruguay In: An. Il Congr. Lat. Amer. Zool., São Paulo, 2:327-335.

Llanos, A. C., 1944. Apreciacones de campo con motivo de una concentracion de roedores en las provincias de Salta y Jujuy. Revta Argent. Zoogeogr., 4:51-57.

Lucero, M. M., 1983. Lista y distribución de aves y mamíferos de la provincia de Tucumán. Minist. de Cultura y Educacion, Rep. Argentina, Miscelanea, 75:1-61.

Massoia, E., 1971. Las ratas nutrias argentinas del genero Holochilus descriptas como Mus braziliensis por Waterhouse (Mammalia - Rodentia - Cricetidae). Rev. Invest. Agropec. Ser. 4 Patol. Animal., 8(5):141-148.

Massoia, E., 1974. Ataques graves de Holochilus y otros roedores a cultivos de caña de azúcar. Inst. Nac. Tecnol. Agropecu (I.N.T.A.) Bol. Inf., (321/24):1-12.

Massoia, E., 1981. El estado sistematico y zoogeografia de Mus brasiliensis DESMAREST ly Holochilus sciureus WAGNER (Mammalia - Rodentia - Cricetidae). Physis 
(B. Aires), Secc. C, 39(97):31-34.

*Miller, L. M. \& Anderson, S., 1978. Bodily proportions of Uruguayan myomorph rodents. Am. Mus. Novit., (2615):1-10.

Ministério do Interior - Sudesul, 1978. Mapeamento da vegetação atual da Região Sul. Porto Alegre. p. 35.

Moojen, J., 1943. Alguns mamíferos colecionados no Nordeste do Brasil. Bolm Mus. Nac. Rio de J., Ser. Zoologia, (5):1-14.

*Morrison-Scott, T. C.S., 1937. An apparently new form of crice tine from British Guiana. Ann. Mag. Nat. Hist., 20(10):535-538.

Osgood, W. H., 1915. New mammals from Brazil and Peru. Field. Mus. Nat. Hist. Publ. Zool. Ser., 10(13):187-198.

Ozanan C. C. A., F., 1969. Notas sobre o rato de cana, "Holochilus sciureus" Wagner, na região do Cariri, Ceará. Rev. Bras. Biol., 29(4):567-570.

${ }^{*}$ Petter, F. \& Tostain, O., 1981. Variabilité de la $3^{\mathrm{e}}$ molaire supérieure d'Holochilus brasiliensis (Rongeurs, Cricetidae). Mammalia, 45(2):257-259.

Riva, R: Vidal, O. R. \& Baro, N. I., 1977. Los cromossomas del genero Holochilus. 2. El cariotipo de $H$. brasiliensis vulpinus. Physis (B. Aires) Ser. C, 36(92):215-218.

Sanborn, C. C., 1929. The land mammals of Uruguay. Field. Mus. Nat. Hist. Publ. Zool. Ser., 17(4):145-165.

Secretaria do Planejamento Municipal de Porto Alegre, 1977. Zoologia do delta do Jacui: Relatório final. Porto Alegre, $76 \mathrm{pp}$.

*Sierra S., B., 1969. Alguns caracteres externs de cricetinos y su relación con el grado de adaptación a la vida acuática (Rodentia). Physis (B. Aires), 28(77):471-486.

Sierra, B. \& Achaval, F., 1971. Ritmos de actividad en Holochilus magnus Hershkovitz. Acta Zool. Lilloana, 28:187-191.

Tate, G. H. H., 1932a. the taxonomic history of the neotropical cricetid genera Holochilus, Nectomys, Scapteromys, Megalomys, Tylomys and Ototylmys. Am. Mus. Novit., 562):1-19.

Thomas, O., 1897. Description of four new South American mammals. Ann. Mag. Nat. Hist., 19(6):218-221.

Thomas, O. 1901. On a collection of mammals from the Kanuku mountains, British Guiana. Ann. Mag. Nat. Hist., 8(7):139-154.

Thomas, O., 1906. Notes on South-American Rodents. Ann. Mag. Nat. Hist., 18(7):441448.

*Thomas, O., 1917. VI - On small mammals from the delta of the Parana. Ann. Mag. Nat. Hist., 20(8):96.

Thomas, O., 1921. Report on the mammalia collected by Mr. Edmund Heller during the Peruvian Expedition of 1915 under the auspices of Yale University and the National Geographical Society. Proc. U. S. Nat. Mus., 58(2333):217-249.

*Thomas, O., 1928. the Goldman-Thomas expedition to Peru. VII. The mamals of the Rio Ucayali. Ann. Mag. Nat. Hist., 2(10):249-265.

Twigg, G. I., 1962. Notes on Holochilus sciureus in British Guiana. J. Mammal., 43(3):369-374.

Twigg, G. 1., 1965a. Studies on Holochilus sciureus berbicensis, a cricetine rodent from coastal region of British Guiana. Proc. Zool. Soc. Lond., 145(2):263-283.

Twigg, G. I., 1965b. Warbles on Holochilus sciureus from the coast of British Guiana. J. Mammal., 46(1):98-100.

Vidal, O. R.; Riva, R. \& Baro, N., 1976. Los cromosomas del genero Holochilus. 1. Polimorfismo en $H$. chacarius Thomas (1906): Physis (B. Aires) Ser. C, 35(90):75-85.

Vidal, O. R. \& Riva, R., 1978. Los cromosomas del genero Holochilus. 3. Inversion, fusion y cromosomas B, nuevos para $H$. chacarius balnearum. Physis (B. Aires) Ser. C, $38(94): 1-5$.

Ximenez, A.; Langguth, A.; Praderi, R., 1972. Lista sistemática de los mamíferos del Uruguay. An. Mus. Hist. Nat. Montevideo. Ser. 2, 7(5):1-49. 
Localização das áreas de coleta para o gênero Holochilus no Rio Grande do Sul.

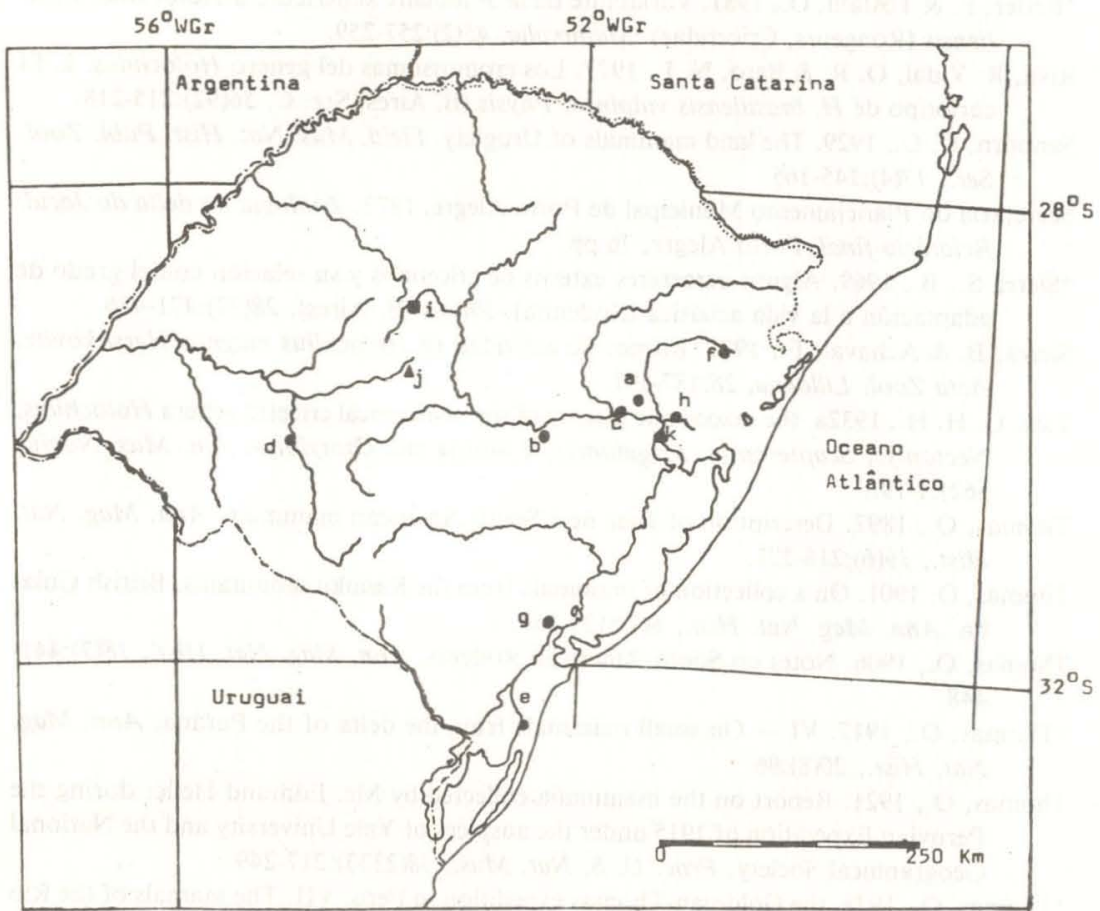

- H. brasiliensis vulpinus: a. Montenegro, b. Pantano Grande, Rio Pardo, c. Triunfo, d. Rosário do Sul, e. Taim, Rio Grande, f. São Francisco de Paula, g. Pelotas, h. Cachoeirinha; $\square$ H. magnus: i. Tupanciretã; $\boldsymbol{\Delta}$ Holochilus sp: j. Santa Maria, k. Delta do Rio Jacuí, Porto Alegre. 
1

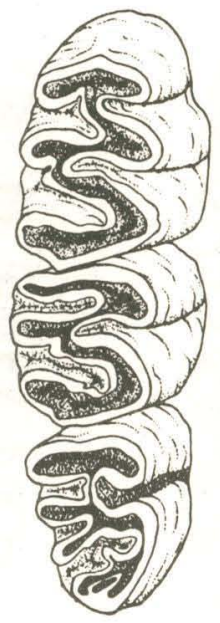

3

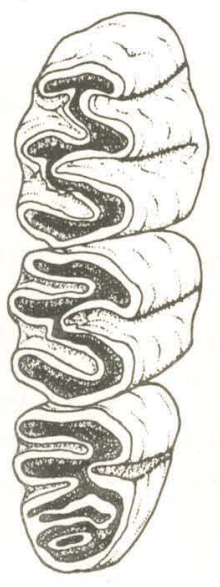

2

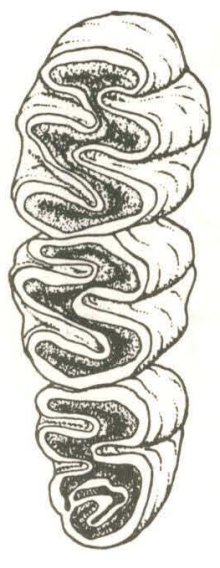

4

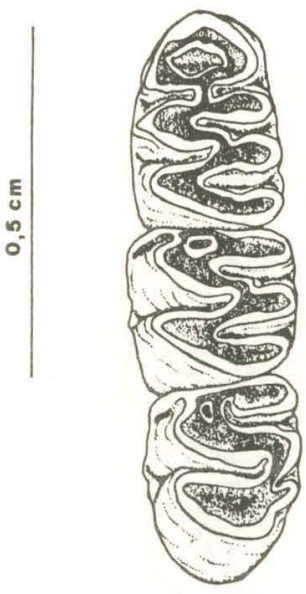

ESTAMPA 1: Séries molares de Holochilus brasiliensis vulpinus (BRANTS, 1827): fig. 1 - MCN.651, superior direita; fig. 2 - MCN.672, superior direita; fig. 3 - MCN.670, superior direita; fig. $4-\mathrm{MCN} .670$, inferior esquerda. 

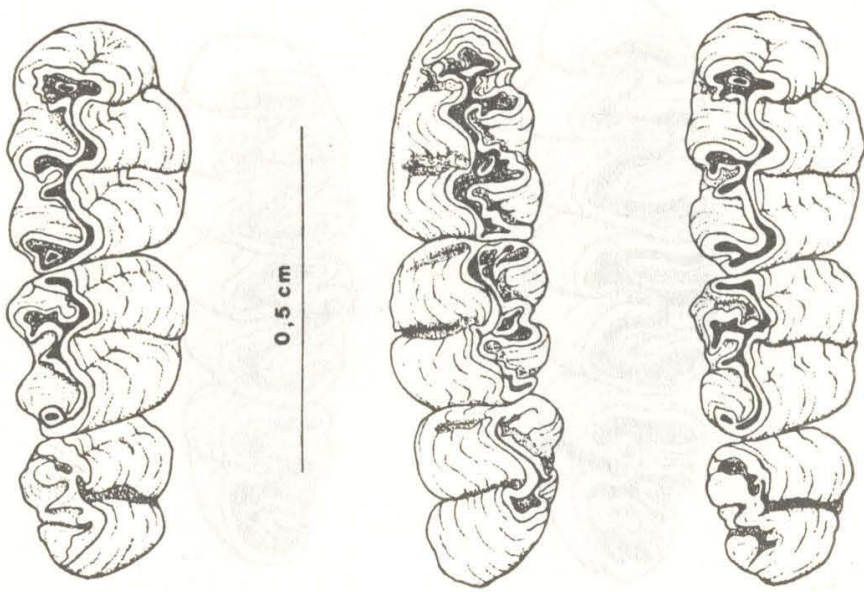

4
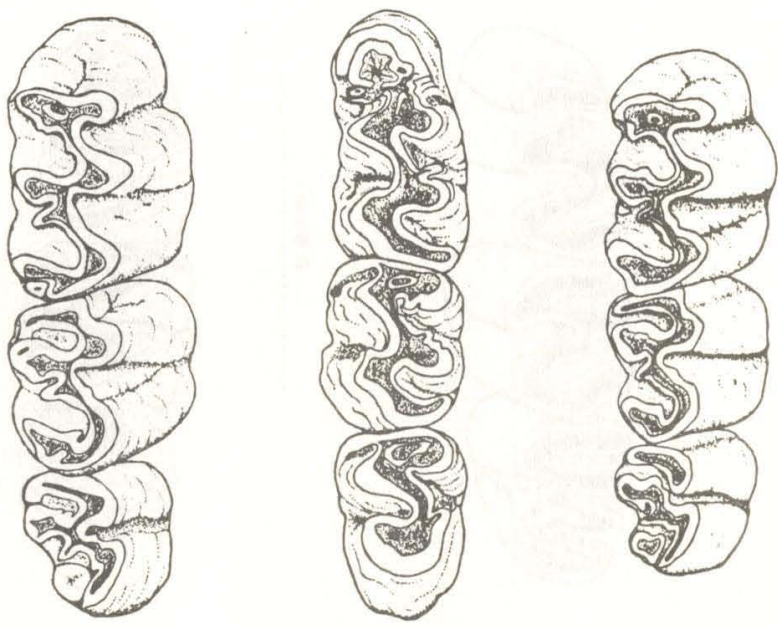

ESTAMPA 2: Séries molares de Holochilus magnusHERSHKOVITZ, 1955: fig. 1 LF.084, superior direita; fig. 2 - LF.084, inferior esquerda; fig. 3 - LF.081, superior direita; fig. 4 - LF.095, superior direita; fig. 5 - LF.095, inferior esquerda; fig. 6 LF.155, superior direita. 